\title{
FROBENIUS RECIPROCITY IN TENSOR CATEGORIES
}

\author{
SHIGERU YAMAGAMI
}

\section{Introduction}

Since the appearance of the celebrated work by V. Jones on index of subfactors, combinatorial structures behind the theory have been exploited extensively. In the original approach of V. Jones, these are described in terms of higher relative commutants of towers of algebras, which turn out to be complete invariants for amenable subfactors by a recent result of S. Popa. Parallel to this approach, A. Ocneanu presented the invariant in a combinatorially more satisfactory way (so called paragroups) based on which he reached his classification result of subfactors of index smaller than 4.

In our previous paper we have described the basics in bimodules to pursue the Ocneanu's approach, where categorical structures underlies behind the whole theory. This point of view has been particularly useful in algebraic studies of Jones index theory ([9], [14], [15], [27]).

This categorical structure turns out to be more fundamental and it deserves separate studies which is the main concern in the present paper.

Since we aim at applications in operator algebras or unitary representation theory, the category considered below are mostly assumed to have *-structure in the sense that morphisms behave like bounded linear operators between Hilbert spaces. Clearly the category of bimodules accomodates such structures and more importantly it admits monoidal structure by taking relative tensor products.

Other than such more or less explicit structures, the category of bimodules (of finite Jones index) bears some metrical information which is directly related to minimal expectations in subfactor theory and has an intimate relation to the notion of connections in paragroups.

By translating some of basic properties of minimal expectations, this distinguished structure, referred to as Frobenius duality in this paper, is organized into a set of axioms, which turns out to be basic ingredients in Frobenius reciprocity. Starting with a Frobenius duality in a monoidal category, we then

Received April 17, 1995; in revised form January 3, 1999. 
develop most of the combinatorical aspects in Jones index theory, such as the Frobenius reciprocity, the additivity of statistical dimensions and the cyclic symmetry of connections, in a purely categorical way.

In recent studies of topological quantum field theory, such a categorical object, which may or may not arise from bimodules, is used to construct topological invariants of 3-manifolds ([3], [4], [22], [29]). There are several good expositions on these aspects of tensor categories ([1], [11], [28]), where the notion of rigidity in tensor categories is combined with braiding structures to produce the relevant ingredients such as quantum traces.

In contrast to these, our Frobenius duality is in some sense a global choice of rigidity, which enables us to separate the notions such as quantum traces from braiding structures.

Thus the present formalism allows us studies of highly non-commutative objects, which are needed as well as useful in the field of original operator algebras: for example, when restricted to monoidal categories realized as unitary representations of compact quantum groups, our Frobenius duality turn out to be modules of Plancherel measures of compact quantum groups in consideration and, with this recognition, we can develop unitary representation theory of compact quantum groups in an ideal way ([32]). We can even give a bimoduletheoretical analogue of crossed products for Roberts actions and all these make it possible to understand the meaning of Ocneanu's characterization of crossed product algebras in its generality ([33], [34]).

After the submission of the present paper, the author found [16], where dimension theory is developped in $C^{*}$-tensor category with the only assumption of rigidity, which strongly suggests the existence and the uniqueness of Frobenius duality in these tensor categories. This is, in fact, the case, which we would like to discuss in a separate paper.

The present article is a partly extended and partly shortened version of preprint, being distributed under the title 'Frobenius reciprocity in monoidal categories', and was completed during the author's visit at the University of Orleans.

He would like to express his hearty thanks to members of the Department of Mathematics, especially to Prof. C. Anantharaman-Delaroche for their warm hospitality and support.

Since the appearance of the draft version of the present paper, there have been compiled several books of related subjects, and we have tried to keep accordance with these, which results in a long delay of updates. The author would like to express his deep gratitude to Prof. Ola Bratteli for the impatience as the correspondent editor. 


\section{Tensor Categories}

By a (complex) tensor category, we shall mean an additive monoidal category with hom-sets given by complex vector spaces and all the relevant operations being linear. Recall that a monoidal category is a category $\mathscr{C}$ with a distinguished object $I$ (called the unit object), a bivariant functor $\otimes: \mathscr{C} \times \mathscr{C} \rightarrow \mathscr{C}$, a natural family of isomorphisms $a=\left\{a_{X, Y, Z}:(X \otimes Y) \otimes Z \rightarrow X \otimes(Y \otimes Z)\right\}$ and natural families of isomorphisms $l=\left\{l_{X}: I \otimes X \rightarrow X\right\}, r=\left\{r_{X}:\right.$ $X \otimes I \rightarrow X\}$ (called the associativity, left unit and right unit constraints respectively) satisfying the pentagon identities and the triangle identities, i.e, the commutativity of the diagrams
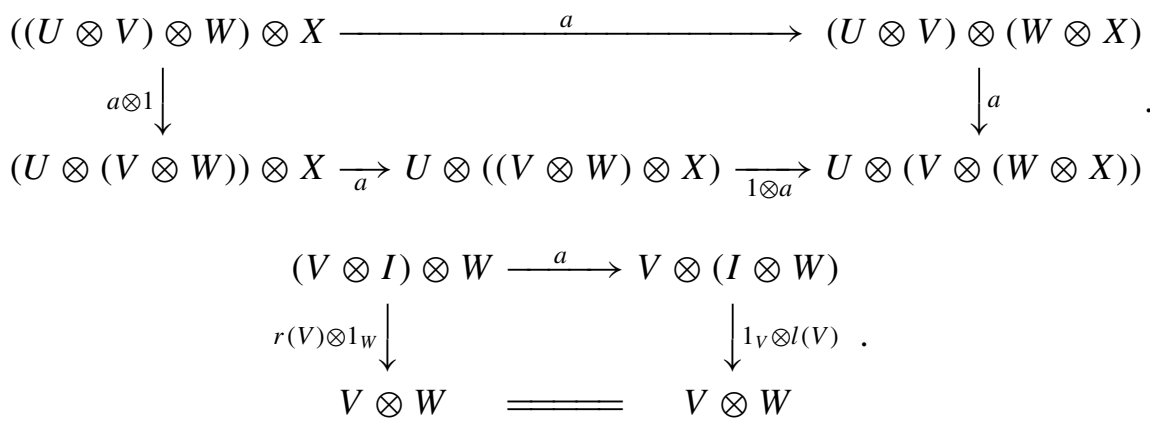

A tensor category is strict if constraints $a, l$ and $r$ are identities $((X \otimes Y) \otimes Z=$ $X \otimes(Y \otimes Z)$ and $I \otimes X=X=X \otimes I$ particularly).

As a variant of tensor categories, we can work with bicategories ([18] $\S 12.6)$ as well: A bicategory consists of a family of categories $\left\{{ }_{A} \mathscr{C}_{B}\right\}$ indexed by double indices in a label set $\mathscr{A}$, where the operation of tensor products is allowed only for objects of the form ${ }_{A} X_{B} \in{ }_{A} \mathscr{C}_{B}$ and ${ }_{B} Y_{C} \in{ }_{B} \mathscr{C}_{C}$. (In the reference [18], $\mathscr{A},{ }_{A} \mathscr{C}_{B}$ and hom-sets in ${ }_{A} \mathscr{C}_{B}$ are referred to as 0-cells, 1-cells and 2-cells respectively.)

By a tensor functor between two tensor categories, we shall mean a monoidal functor which respects the linear structures: a tensor functor consists of a functor $F: \mathscr{C} \rightarrow \mathscr{D}$ between tensor categories $\mathscr{C}$ and $\mathscr{D}$ together with a natural family of isomorphisms $\left\{m_{X, Y}: F(X) \otimes F(Y) \rightarrow F(X \otimes Y)\right\}$ satisfying $F(I) \cong I$ and

Let $F, G: \mathscr{C} \rightarrow \mathscr{D}$ be two tensor functors. A natural transformation $\left\{\varphi_{X}: F(X) \rightarrow G(X)\right\}$ is monoidal if it satisfies

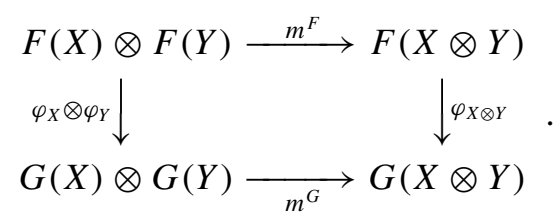


A natural monoidal transformation is called a monoidal natural equivalence if $\varphi_{X}$ is an isomorphism for each $X$.

A monoidal functor $F: \mathscr{C} \rightarrow \mathscr{C}^{\prime}$ is called a monoidal equivalence if there are a monoidal functor $G: \mathscr{C}^{\prime} \rightarrow \mathscr{C}$ and two monoidal natural equivalences

$$
\varphi: F G \cong \operatorname{id}_{\mathscr{C}^{\prime}}, \quad \psi: G F \cong \operatorname{id}_{\mathscr{C}} .
$$

Two tensor categories $\mathscr{C}, \mathscr{C}^{\prime}$ are said to be equivalent if there is a monoidal equivalence $F: \mathscr{C} \rightarrow \mathscr{C}^{\prime}$.

By a duality in a tensor category $\mathscr{C}$, we mean a contravariant functor $X \mapsto X^{*}, \operatorname{Hom}(X, Y) \ni f \mapsto{ }^{t} f \in \operatorname{Hom}\left(Y^{*}, X^{*}\right)$ (the map $f \mapsto{ }^{t} f$ being linear) with natural families of isomorphisms $\left\{c_{X, Y}: Y^{*} \otimes X^{*} \rightarrow(X \otimes Y)^{*}\right\}$ (antimultiplicativity) and $\left\{d_{X}: X \rightarrow\left(X^{*}\right)^{*}\right\}$ satisfying

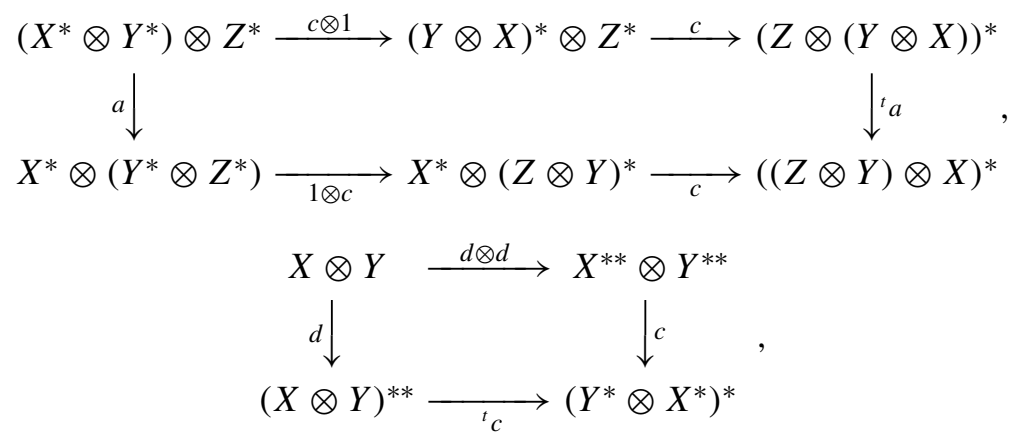

and ${ }^{t} d_{X}=d_{V X^{*}}^{-1}: X^{* * *} \rightarrow X^{*}$. (The naturality means ${ }^{t}(f \otimes g) \stackrel{c}{\sim}{ }^{t} g \otimes{ }^{t} f$, $f \stackrel{d}{\sim}{ }^{t}\left({ }^{t} f\right)$.)

We remark here that the operation $\left(X \mapsto X^{*}, f \mapsto{ }^{t} f\right)$ together with $c$ satisfying the antimultiplicativity is an antimonoidal functor and we see that $f \mapsto{ }^{t}\left({ }^{t} f\right)$ gives a tensor functor with the multiplicativity

$$
{ }^{t} c^{-1} \circ c: X^{* *} \otimes Y^{* *} \rightarrow\left(Y^{*} \otimes X^{*}\right)^{*} \rightarrow(X \otimes Y)^{* *} .
$$

The duality isomorphism $d$ is then nothing but a monoidal natural equivalence between the tensor functors $f \mapsto{ }^{t}\left({ }^{t} f\right)$ and $f \mapsto f$.

Remark. Although it is common in linear algebras, the different notations for objects and morphisms would be awkward from esthetic points of view; the preferable one would be $f^{*}$. This reasonable notation, however, conflicts with the notation taking adjoints of operators on Hilbert spaces, if one deals with tensor categories originated from operator algebras.

Let $\mathscr{C}$ and $\mathscr{D}$ be tensor categories with duality. A monoidal functor $F: \mathscr{C} \rightarrow$ $\mathscr{D}$ preserves duality, definition, if there is a natural family $\left\{s_{X}: F\left(X^{*}\right) \rightarrow\right.$ 
$\left.F(X)^{*}\right\}$ of isomorphisms in $\mathscr{D}$ satisfying

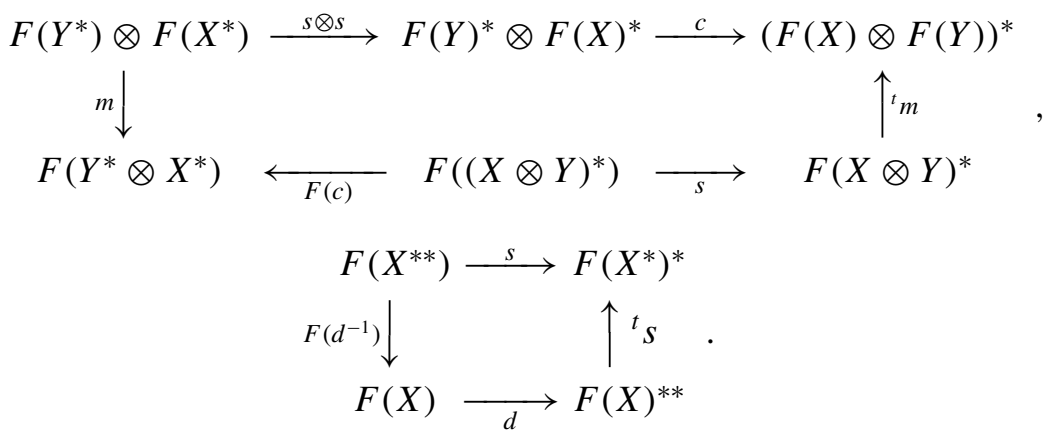

Two tensor categories with duality is said to be equivalent if we can choose functors $F, G$ of monoidal equivalence so that they preserves duality.

As a result of the celebrated coherence theorem of S. MacLane ([17]), we know that any tensor category is equivalent to a strict one and here is an analogue for tensor categories with duality, which can be proved by an obvious modification of the method in [11, Chap. XI.5].

THEOREM 1.1. Any tensor category with duality is equivalent to a strict one.

This theorem gives rise to the coherence theorem involving duality, which allows us to omit the cumbersome isomorphisms $a, l, r c$ and $d$ in formulas, or we may just work with strict tensor categories.

Here are some typical examples of tensor categories with duality.

Example 1.2. Let $\mathscr{A}$ be a family of von Neumann algebras. Recall that an $A-B(A, B \in \mathscr{A})$ bimodule is a Hilbert space $X$ together with normal representations of $A$ and $B$ on $X$ in a bimodule fashion.

For an $A-B$ bimodule $X$, the dual bimodule $X^{*}$ (which is a $B-A$ bimodule) is the dual Hilbert space $X^{*}$ with the actions

$$
b \xi^{*} a=\left(a^{*} \xi b^{*}\right)^{*}, \quad \xi \in X, \quad a \in A, \quad b \in B,
$$

where $\xi^{*}$ denotes the linear functional on $X$ associated to $\xi \in X$ through the inner product. The tensor product of an $A-B$ bimodule $X$ and a $B-C$ bimodule $Y$ is defined by the relative tensor product $X \otimes^{B} Y$ with the obvious $A$ - $C$ action from the outside (see [26], [31] for details on relative tesnsor products). The associativity constraint $\left(X \otimes^{B} Y\right) \otimes^{C} Z \rightarrow X \otimes^{B}\left(Y \otimes^{C} Z\right)$ is given by the natural isomorphism of relative tensor products. (In the notation of [31], this is realized as $\left(\xi \otimes \varphi^{-1 / 2} \otimes \eta\right) \otimes \psi^{-1 / 2} \otimes \zeta \mapsto \xi \otimes \varphi^{-1 / 2} \otimes\left(\eta \otimes \supset^{-1 / 2} \otimes \zeta\right)$.) For $A \in \mathscr{A}$, the standard representation ${ }_{A} L^{2}(A)_{A}$ provides the unit object.

With this structure, the category of bimodules with actiong algebras in $\mathscr{A}$ becomes to be a bicategory (over $\mathscr{A}$ ). 
EXAmPLE 1.3. Let $G=(\mathscr{M}, h)$ be a quantum group in the sense that $\mathscr{M}$ is a Hopf algebra with an invertible element $h \in \mathscr{M}$ satisfying $\Delta(h)=h \otimes h$, $\sigma(h)=h^{-1}, \sigma^{2}=A d h^{2}(\sigma$ denotes the antipode of $\mathscr{M})$. The associated antiautomorphism $\tau$ is defined by $\tau=\sigma \circ A d h^{-1}=A d h^{-1} \circ \sigma$. Note that the $q$-deformation of the universal enveloping algebra of a simple Lie algebra belongs to this class.

The category $\mathscr{R}(G)$ of finite-dimensional $\mathscr{M}$-modules has the structure of tensor category in the following way: for $\mathscr{M}$-modules $V, W$, the ordinary tensor product $V \otimes W$ becomes an $\mathscr{M}$-module by $x_{V \otimes W}=\Delta(x)_{V \otimes W}, x \in \mathscr{M}$ and the dual object of $V$ is given by the dual space $V^{*}$ together with the action defined by

$$
\left\langle x v^{*}, v\right\rangle=\left\langle v^{*}, \tau(x) v\right\rangle, \quad x \in \mathscr{M}, \quad v \in V, \quad v^{*} \in V^{*} .
$$

The unit object in $\mathscr{R}(G)$ is the one-dimensional representation of $\mathscr{M}$ given by the counit. Note that the obvious isomorphism $V^{* *} \cong V$ as vector spaces is $\mathscr{M}$-linear due to the use of $\tau$ instead of $\sigma$ and gives rise to the duality isomorphism $d_{V}$ in the tensor category $\mathscr{R}(G)$.

EXAMPLE 1.4 ([10]). Let $\Gamma$ be a locally compact second countable group and denote by $C_{0}(\Gamma)$ the commutative $C^{*}$-algebra of continuous functions on $\Gamma$ vanishing at infinity. Let $V$ be a $C_{0}(\Gamma)$-module, i.e., $V$ is a Hilbert space on which $C_{0}(\Gamma)$ is represented as a $C^{*}$-algebra. Let $H$ and $K$ be two compact subgroups in $\Gamma$. A $C_{0}(\Gamma)$-module $V$ is called an $H$ - $K$ bundle over $\Gamma$ if $V$ admits an $H$ - $K$ action (i.e., a commuting pair of left $H$ - and right $K$ - unitary representations) satisfying

$$
h(f v)=(h . f)(h v), \quad(f v) k=(f . k)(v k), \quad \text { for } \quad \begin{aligned}
& f \in C_{0}(\Gamma), \quad v \in V, \\
& h \in H, \quad k \in K .
\end{aligned}
$$

Here $h . f$ and $f . k$ denote left and right translations of $f$. An $H-K$ bundle $V$ is often expressed as $V={ }_{H} V_{K}$ to visualize the actiong subgroups.

The category of such bundles for various pairs of subgroups has the structure of bicategory with duality: For $V={ }_{H} V_{K}$, the dual bundle $V^{*}={ }_{K} V_{H}$ is defined to be the dual Hilbert space of $V$ with $C_{0}(\Gamma)$ - and $K-H$ actions given by

$$
f v^{*}=\left(f^{*} v\right)^{*}, \quad k v^{*} h=\left(h^{-1} v k^{-1}\right)^{*}
$$

with $f^{*}(g)=\overline{f\left(g^{-1}\right)}$.

For $V={ }_{G} V_{H}$ and $W={ }_{H} W_{K}$, let $V \otimes_{H} W$ be the closed subspace of $V \otimes W$ consisting of invariant vectors under the unitary representation $\pi$ of $H$ defined by

$$
\pi(h)(v \otimes w)=\left(v h^{-1}\right) \otimes(h w)
$$


with an obvious $G$ - $K$ action from outside. The action of $C_{0}(\Gamma)$ on $V \otimes_{N} W$ is given by restricting the action of $C_{0}(\Gamma)$ on $V \otimes W$, where $C_{0}(\Gamma)$ acts on $V \otimes W$ by dualizing the map $\Gamma \times \Gamma \ni\left(g_{1}, g_{2}\right) \ni g_{1} g_{2} \in \Gamma$.

\section{Frobenius Duality}

In this section, we introduce a rigid structure of duality, which plays the central role in what follows.

DeFinition 2.1. By a Frobenius duality in a (strict) tensor category, we mean a strict duality together with a family of morphisms $\left\{\epsilon_{X}: X \otimes X^{*} \rightarrow\right.$ $I\}_{X \in \text { Object }}$ satisfying the following conditions.

(i) (Multiplicativity)

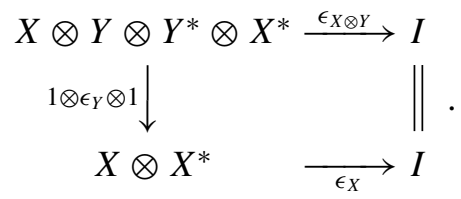

(ii) (Naturality) For a morphism $f: X \rightarrow Y$ in $\mathscr{C}$,

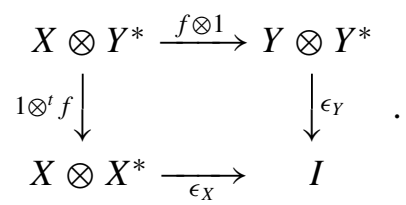

(iii) (Faithfulness) The map

$$
\operatorname{Hom}(X, Y) \ni f \mapsto \epsilon_{Y} \circ(f \otimes 1) \in \operatorname{Hom}\left(X \otimes Y^{*}, I\right)
$$

is injective for $X, Y \in \operatorname{Object}(\mathscr{C})$.

(iv) (Balancedness) For a morphism $f \in \operatorname{End}(X)$, we have

$$
\epsilon_{X}(f \otimes 1)^{t} \epsilon_{X}=\epsilon_{X^{*}}(1 \otimes f)^{t} \epsilon_{X^{*}}
$$

REMARK. The definition has the meaning even for bicategories except for the axim of balancedness: For an object $X$ in ${ }_{A} \mathscr{C}_{B}, \epsilon_{X}{ }^{t} \epsilon_{X}$ and $\epsilon_{X^{*}}{ }^{t} \epsilon_{X^{*}}$ belong to endomorphisms of different unit objects $I_{A}$ and $I_{B}$. The easiest way to compare these is to assume the simplicity of unit objects, which allows us to introduce the quantum trace $\langle\cdot\rangle_{X}$ below as a linear functional on $\operatorname{End}(X)$. Then the balancedness is formulated as $\langle f\rangle_{X}=\left\langle{ }^{t} f\right\rangle_{X}$.

ExAmPLE 2.2. The category of bimodules of finite Jones index admits the canonical Frobenius duality defined by

$$
{ }^{t} \epsilon_{X}\left(\varphi^{1 / 2}\right)=[X]^{1 / 4}(\varphi \circ E)^{1 / 2} .
$$


Here $\varphi^{1 / 2}$ refers to the (canonical) GNS-vector corresponding to a normal positive linear functional $\varphi$ on $A, E: \operatorname{End}\left(X_{B}\right) \rightarrow A$ the minimal expectation, and $[X]$ the minimal index of the inclusion $A \subset B^{\prime} \equiv \operatorname{End}\left(X_{B}\right)(X$ is assumed to be an $A$ - $B$ bimodule). Here $X \otimes^{B} X^{*}$ is naturally identified with $L^{2}\left(B^{\prime}\right)$. For the proof of the required properties of Frobenius duality, we refer to [30].

ExAmPLE 2.3. The Tannaka dual $\mathscr{R}(G)$ in Example 1.3 admits the canonical Frobenius duality defined by

$$
\epsilon_{V}\left(v \otimes v^{*}\right)=\left\langle h v, v^{*}\right\rangle, \quad v \in V, \quad v^{*} \in V^{*} .
$$

Here the (representation space of) trivial representation is identified with C.

EXAmPLE 2.4. Let $\Gamma$ be a discrete group and $H, K \subset \Gamma$ be finite subgroups. Then any $H$ - $K$ bundle $V$ can be decomposed as $V=\oplus_{g \in \mathbf{s}(V)} V_{g}(\mathbf{s}(V) \subset \Gamma$ denotes the support of $V$ ). In particular, the trivial $H-H$ bundle is given by ${ }_{H} I_{H}=\oplus_{h \in H} \mathrm{Ch}$. We can define a morphism ${ }^{t} \epsilon_{V}:{ }_{H} I_{H} \rightarrow{ }_{H} V \otimes V_{H}^{*}$ by extending the map

$$
\mathrm{C} e \ni e \mapsto \bigoplus_{\dot{g} \in \mathbf{s}(V) / K} \sum_{i=1}^{\operatorname{dim} V_{g}} v_{g}^{(i)} \otimes_{K} v_{g}^{(i)^{*}} \in\left(V \otimes_{K} V^{*}\right)_{e} \subset V \otimes_{H} V^{*}
$$

by $H$ - $H$ equivariance. Here $\left\{v_{g}^{(i)}\right\}_{1 \leq i \leq \operatorname{dim} V_{g}}$ denotes an orthogonal basis in $V_{g}$ and $\dot{g} \in \mathbf{s}(V)$ runs through a representative of $\mathbf{s}(V) / K$. It is then easy to check that these give Frobenius duality for the bicategory considered in Example 1.4.

For the time being, we fix a (strict) tensor category $\mathscr{C}$ with Frobenius duality $\left\{\epsilon_{X}\right\}$.

LEMMA 2.5 .

(i) For $X=I, \epsilon_{X}$ is given by the unit constaint, i.e., the identity morphism as the tensor category being assumed strict.

(ii) For any object $X,\left(\epsilon_{X} \otimes 1_{X}\right)\left(1_{X} \otimes{ }^{t} \epsilon_{X^{*}}\right)=1_{X}=\left(1_{X} \otimes \epsilon_{X^{*}}\right)\left({ }^{t} \epsilon_{X} \otimes 1_{X}\right)$.

Proof. (i) follows from the multiplicativity and the faithfulness.

(ii) Let $f=\left(\epsilon_{X} \otimes 1_{X}\right)\left(1_{X} \otimes{ }^{t} \epsilon_{X^{*}}\right) \in \operatorname{End}(X)$ and calculate as follows:

$$
\begin{aligned}
\epsilon_{X^{*}}\left(1_{X^{*}} \otimes f\right) & =\epsilon_{X^{*} \otimes X}\left(1_{X^{*} \otimes X} \otimes{ }^{t} \epsilon_{X^{*}}\right) & & \text { (use the multiplicativity) } \\
& =\epsilon_{I}\left(\epsilon_{X^{*}} \otimes 1_{I}\right) & & \text { (use the naturality) } \\
& =\epsilon_{X^{*}} & & \text { (use (i) already checked). }
\end{aligned}
$$

From the faithfulness axiom, we conclude that $f=1_{X}$. 
The identities in (ii) are graphically expressed as Fig. 1 (morphisms are drawn from up to down) and called hook identities by the obvious reason.

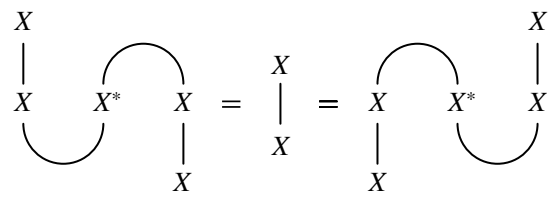

FiguRe 1

REMARK. The existence of a pair of morphisms fulfilling hook identities is called the rigidity in literatures, which enables us to associate, up to natural isomorphisms, a weaker form of duality discussed in the previous section (see [1], [11] for more complete accounts).

In this sense, our Frobenius duality is a global form of rigidity and their differences can be described certain cohomological obstructions (cf. [35]).

Definition 2.6. Given an object $X$ in a tensor category $\mathscr{C}$ with Frobenius duality, we introduce the quantum trace $\langle\cdot\rangle_{X}$ as an $\operatorname{End}(I)$-valued linear functional on $\operatorname{End}(X)$ defined by

$$
\langle f\rangle_{X}=\epsilon_{X}\left(f \otimes 1_{X^{*}}\right)^{t} \epsilon_{X} .
$$

REMARK. As can be easily seen and well-known, $\operatorname{End}(I)$ is a commutative algebra. When the unit object $I$ is simple, End $(I)$ is identified with the complex number field and quantum traces are linear functionals.

Proposition 2.7.

(i) For $S \in \operatorname{Hom}(X, Y)$ and $T \in \operatorname{Hom}(Y, X)$, we have

$$
\langle S T\rangle_{Y}=\langle T S\rangle_{X} .
$$

(ii) For $S \in \operatorname{End}(X)$ and $T \in \operatorname{End}(Y)$, we have

$$
\langle S \otimes T\rangle_{X \otimes Y}=\langle S\rangle_{X}\langle T\rangle_{Y}
$$

(iii) and

$$
\left\langle{ }^{t} S\right\rangle_{X}={ }^{t}\langle S\rangle_{X} .
$$

Proof. (i) We first consider the case $X=Y$. Then the trace property is checked by

$$
\begin{aligned}
\epsilon_{X}(S T \otimes 1)^{t} \epsilon_{X} & =\epsilon_{X}(S \otimes 1)^{t}\left(\epsilon_{X}\left(1 \otimes{ }^{t} T\right)\right)=\epsilon_{X}\left(1 \otimes{ }^{t} S\right)^{t}\left(\epsilon_{X}(T \otimes 1)\right) \\
& =\epsilon_{X}\left(1 \otimes{ }^{t} S^{t} T\right)^{t} \epsilon_{X}=\epsilon_{X}(T S \otimes 1)^{t} \epsilon_{X} .
\end{aligned}
$$


To deal with the general case, let $Z$ be a direct sum of $X$ and $Y$ with injections $i_{X}: X \rightarrow Z, i_{Y}: Y \rightarrow Z$ and projections $j_{X}: Z \rightarrow X, j_{Y}: Z \rightarrow Y$. Then we have the expression

$$
\begin{aligned}
\langle T S\rangle_{X} & =\epsilon_{X}\left(T S \otimes{ }^{t} i_{X}{ }^{t} j_{X}\right)^{t} \epsilon_{X}=\epsilon_{X}\left(1 \otimes{ }^{t} i_{X}\right)(T S \otimes 1)\left(1 \otimes{ }^{t} j_{X}\right)^{t} \epsilon_{X} \\
& =\epsilon_{Z}\left(i_{X} \otimes 1\right)(T S \otimes 1)\left(j_{X} \otimes 1\right)^{t} \epsilon_{Z}=\left\langle i_{X} T S j_{X}\right\rangle_{Z}=\left\langle i_{X} T j_{Y} i_{Y} S j_{X}\right\rangle_{Z} .
\end{aligned}
$$

If we apply the trace property of $\langle\cdot\rangle_{Z}$, the last expression takes the form $\left\langle i_{Y} S j_{X} i_{X} T j_{Y}\right\rangle_{Z}$, which turns out to be equal to $\langle S T\rangle_{Y}$ by reversing the computation.

Definition 2.8. For an object $X$ in a tensor category with Frobenius duality, define its quantum dimension by

$$
d(X)=\left\langle 1_{X}\right\rangle_{X} .
$$

\section{REMARK.}

(i) Categorical definitions of quantum dimension as well as quntum trace are considered in literatures mainly from the viewpoint of topological quantum field theory, where the definition is derived from rigidity, a weaker notion of Frobenius duality, together with the use of braiding structures on tensor categories ([1], [11], [28]). We here introduced quantum dimension even for highly non-commutative objects, which turns out to be useful in categorical studies of harmonic analysis of subfactors and group representation theory.

(ii) In the case of bimodules of finite index, $d(X)$ coincides with the square root of the minimal index of the inclusion $A \subset \operatorname{End}\left(X_{B}\right)$ ([8]) or the statistical dimension in sector theory ([14]).

(iii) In the Tannaka dual of a quantum group, the quantum dimension of a representation $V$ is given by $d(V)=\operatorname{trace}_{V}\left(h^{2}\right)$, where trace denotes the ordinary trace for linear operators.

Proposition 2.9. The dimension function has the following properties:

(i) The dimension of the unit object I is the unit in the commutative algebra $\operatorname{End}(I)$.

(ii) $d(X \oplus Y)=d(X)+d(Y)$.

(iii) $d(X \otimes Y)=d(X) d(Y)$.

(iv) If $X \cong Y$, then $d(X)=d(Y)$.

Proof. (i) is obvious by Lemma 2.5 (i). (iv) results from Proposition 2.7 while (iii) is a direct consequence of the multiplicativity. To check (ii), we 
apply the argument in the proof of Proposition 2.7 to get

$$
d(X \oplus Y)=\epsilon_{Z}{ }^{t} \epsilon_{Z}=\epsilon_{X}{ }^{t} \epsilon_{X}+\epsilon_{Y}{ }^{t} \epsilon_{Y}=d(X)+d(Y) .
$$

\section{Cyclic Tensor Products}

In what follows we shall work with a strict tensor category $\mathscr{C}$ with Frobenius duality $\left\{\epsilon_{X}\right\}$.

Definition 3.1. Let $X, Y$ and $Z$ be objects in $\mathscr{C}$. Given a morphism $f$ in $\operatorname{Hom}(X \otimes Y, Z)$, its left and right Frobenius transforms are defined by

$$
g=\left(1_{X^{*}} \otimes f\right)\left({ }^{t} \epsilon_{X^{*}} \otimes 1_{Y}\right), \quad h=\left(f \otimes 1_{Y^{*}}\right)\left(1_{X} \otimes{ }^{t} \epsilon_{Y}\right) .
$$

Proposition 3.2 (Frobenius Reciprocity). Frobenius transforms are isomorphisms and their inverses are given by

$$
\left(\epsilon_{X} \otimes 1_{Z}\right)\left(1_{X} \otimes g\right)=f=\left(1_{Z} \otimes \epsilon_{Y^{*}}\right)\left(h \otimes 1_{Y}\right) .
$$

LeMma 3.3. A repetition of left (right) Frobenius transforms is again a left (right) Frobenius transform.

Definition 3.4. Given an object $X$ in $\mathscr{C}$, the vector space $H(X)=$ $\operatorname{Hom}(I, X)$ is called the cyclic tensor product.

Let $X, Y$ be objects in $\mathscr{C}$ and $L: \operatorname{Hom}(X, Y) \rightarrow H\left(X^{*} \otimes Y\right), R$ : $\operatorname{Hom}(X, Y) \rightarrow H\left(Y \otimes X^{*}\right)$ be special cases of Frobenius transform:

$$
L(T)=\left(1_{X^{*}} \otimes T\right)^{t} \epsilon_{X^{*}}, \quad R(T)=\left(T \otimes 1_{X^{*}}\right)^{t} \epsilon_{X} .
$$

LEMMA 3.5. Both of $L^{-1} R$ and $R^{-1} L$ are given by the transposition involved in the duality functor.

Proof. For $T \in \operatorname{Hom}(X, Y)$,

$$
L\left({ }^{t} T\right)=\left(1_{Y} \otimes{ }^{t} T\right)^{t} \epsilon_{Y}=(T \otimes 1)^{t} \epsilon_{X}=R(T) .
$$

Thus $L^{-1} R(T)={ }^{t} T$ and similarly for $R^{-1} L$.

Combining the isomorphisms in the above lemma, we obtain the commutative diagram

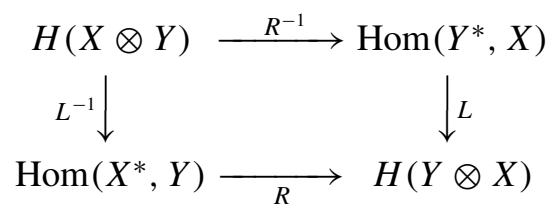


and the isomorphism $C_{X, Y}: H(X \otimes Y) \rightarrow H(Y \otimes X)$, called the cyclic transform. According to two routes (upper and lower ones) in the diagram, we have two expressions for the cyclic transform:

$C_{X, Y}(T)=\left(1_{Y X} \otimes \epsilon_{Y}\right)\left(1_{Y} \otimes T \otimes 1_{Y^{*}}\right)^{t} \epsilon_{Y}=\left(\epsilon_{X^{*}} \otimes 1_{Y X}\right)\left(1_{X^{*}} \otimes T \otimes 1_{X}\right)^{t} \epsilon_{X^{*}}$.

We present another formula which turns out to be useful in later computations:

Lemma 3.6. Take $X, Y$ be objects in $\mathscr{C}$ and $T \in H(X \otimes Y)$. Then

$$
C_{X, Y}(T)=\left(1_{Y} \otimes{ }^{t} T \otimes 1_{X}\right)\left({ }^{t} \epsilon_{Y} \otimes{ }^{t} \epsilon_{X^{*}}\right) .
$$

In the graphical presentation, this is expressed by Fig 2.

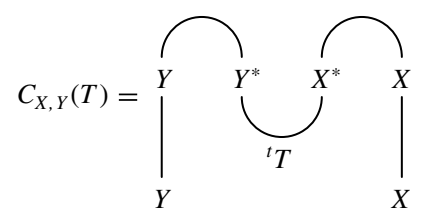

FIGURE 2

Proof. By the naturality, we have $\left(1_{Y} \otimes T \otimes 1_{Y^{*}}\right)^{t} \epsilon_{Y}=\left({ }^{t}\left(T \otimes 1_{Y^{*}}\right) \otimes\right.$ $\left.1_{X Y Y^{*}}\right)^{t} \epsilon_{Y Y^{*} X^{*}}$, which is used in $C_{X, Y}(T)=\left(1_{Y X} \otimes \epsilon_{Y}\right)\left(1_{Y} \otimes T \otimes 1_{Y^{*}}\right)^{t} \epsilon_{Y}$ and calculate as Fig 3.

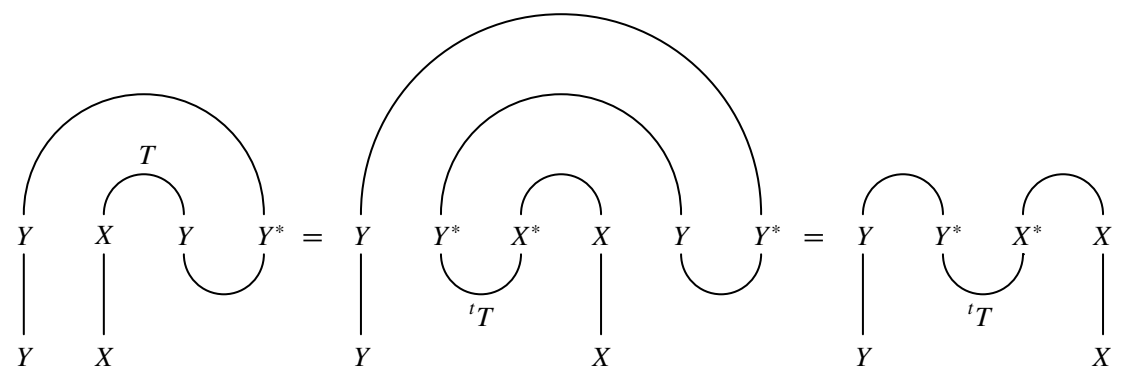

FIGURE 3

Iterations of cyclic transforms bear a kind of coherence, which was first recognized by A. Ocneanu in [22] as $S_{4}$-symmetry and is refered to as cyclic symmetry below (the $S_{4}$-symmetry is a combination of the cyclic symmetry and the conjugation symmetry discussed below). 
Proposition 3.7 (Cyclic Symmetry).

(i) Cyclic transforms are natural in its variables.

(ii) $C_{X, I}=\operatorname{id}_{H(X)}=C_{I, X}$.

(iii) $C_{Y, X} \circ C_{X, Y}=\operatorname{id}_{H(X \otimes Y)}$.

(iv) $C_{Y, Z \otimes X} \circ C_{X, Y \otimes Z}=C_{X \otimes Y, Z}$.

Proof. (i) We need to show the following diagram commute:

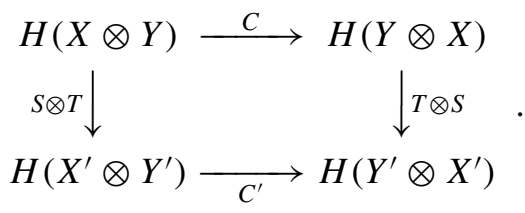

This is checked by

$$
\begin{aligned}
(T \otimes S) C_{X, Y}(U) & =(T \otimes S)\left(1_{Y} \otimes{ }^{t} U \otimes 1_{X}\right)\left({ }^{t} \epsilon_{Y} \otimes{ }^{t} \epsilon_{X^{*}}\right) \\
& =\left(1_{Y^{\prime}} \otimes{ }^{t} U \otimes 1_{X^{\prime}}\right)\left(T \otimes 1_{Y^{*} X^{*}} \otimes S\right)\left({ }^{t} \epsilon_{Y} \otimes{ }^{t} \epsilon_{X^{*}}\right) \\
& =\left(1_{Y^{\prime}} \otimes{ }^{t} U \otimes 1_{X^{\prime}}\right)\left(1_{Y^{\prime}} \otimes\left({ }^{t} T \otimes{ }^{t} S\right) \otimes 1_{X^{\prime}}\right)\left({ }^{t} \epsilon_{Y^{\prime}} \otimes{ }^{t} \epsilon_{X^{*}}\right) \\
& =C_{X^{\prime}, Y^{\prime}}((T \otimes S) U) .
\end{aligned}
$$

(ii) Put $Y=I$ in Lemma 2.5. Then

$$
C_{X, I}(T)=\left({ }^{t} T \otimes 1_{X}\right)^{t} \epsilon_{X^{*}}=\left(1_{I} \otimes T\right)^{t} \epsilon_{I}=T .
$$

(iii) follows from (ii) and (iv) by taking $Z=I$.

(iv) Let $T \in H(X \otimes Y \otimes Z)$. Repeating definitions, we have

$C_{Y, Z X} \circ C_{X, Y Z}(T)$

$$
\begin{aligned}
& =\left(1_{Z X Y} \otimes{ }^{t t} \epsilon_{Z X}\right)\left(1_{Z X Y Z X} \otimes \epsilon_{Y Z} \otimes 1_{X^{*} Z^{*}}\right) \\
& \quad\left(1_{Z X Y Z} \otimes T \otimes 1_{Z^{*} Y^{*} X^{*} Z^{*}}\right)\left(1_{Z X} \otimes{ }^{t} \epsilon_{Y Z} \otimes 1_{X^{*} Z^{*}}\right)^{t} \epsilon_{Z X} \\
& =\left(1_{Z X Y} \otimes \epsilon_{Z}\right)\left(1_{Z X Y Z} \otimes \epsilon_{X} \otimes 1_{Z^{*}}\right)\left(1_{Z X Y Z X} \otimes \epsilon_{Y Z} \otimes 1_{X^{*} Z^{*}}\right) \\
& \quad\left(1_{Z X Y Z} \otimes T \otimes 1_{Z^{*} Y^{*} X^{*} Z^{*}}\right)\left(1_{Z X} \otimes{ }^{t} \epsilon_{Y Z} \otimes 1_{X^{*} Z^{*}}\right) \\
& \quad\left(1_{Z} \otimes{ }^{t} \epsilon_{X} \otimes 1_{Z^{*}}\right)^{t} \epsilon_{Z} .
\end{aligned}
$$

Thus the problem is reduced to show that

$\left(1_{X Y Z} \otimes \epsilon_{X}\right)\left(1_{X Y Z X} \otimes \epsilon_{Y Z} \otimes 1_{X^{*}}\right)\left(1_{X Y Z} \otimes T \otimes 1_{Z^{*} Y^{*} X^{*}}\right)\left(1_{X} \otimes{ }^{t} \epsilon_{Y Z} \otimes 1_{X^{*}}\right)^{t} \epsilon_{X}$ $=T$.

This last equation follows from Lemma 3.3 because $\left(1_{X} \otimes{ }^{t} \epsilon_{Y Z} \otimes 1_{X^{*}}\right)^{t} \epsilon_{X}=$ ${ }^{t} \epsilon_{X Y Z}$ by multiplicativity of Frobenius duality. 
REMARK. Up to this point, we do not use the neutrality axiom of Frobenius duality and the results are valid for bicategories as well.

For an object $X$ in $\mathscr{C}$, define an $\operatorname{End}(I)$-valued bilinear form $\langle$,$\rangle on$ $H(X) \times H\left(X^{*}\right)$ by

$$
\langle S, T\rangle={ }^{t} S T=\epsilon_{X}(S \otimes T) .
$$

The pairing $\langle$,$\rangle is symmetric in the sense that { }^{t}\langle S, T\rangle=\langle T, S\rangle$.

LEMma 3.8. For $S \in H(X \otimes Y)$ and $T \in H\left(Y^{*} \otimes X^{*}\right)$, let $\widetilde{S} \in H(Y \otimes X)$ and $\widetilde{T} \in H\left(X^{*} \otimes Y^{*}\right)$ be their cyclic transforms. Denote by $S_{X^{*}}: X^{*} \rightarrow Y$ the associated Frobenius transform and similarly for others. Then we have

$$
\langle S, T\rangle=\left\langle{ }^{t} S_{X^{*}} T_{X}\right\rangle_{X}=\left\langle S_{X^{*}}{ }^{t} T_{X}\right\rangle_{Y}=\langle\widetilde{S}, \widetilde{T}\rangle .
$$

Proof. By the definition of pairing,

$$
\langle S, T\rangle={ }^{t} S T=\epsilon_{X}\left({ }^{t} S_{X^{*}} \otimes 1\right)\left(T_{X} \otimes 1\right)^{t} \epsilon_{X}=\tau_{X}\left({ }^{t} S_{X^{*}} T_{X}\right),
$$

which gives the first relation.

Similarly, we have

$$
\langle\widetilde{S}, \widetilde{T}\rangle=\tau_{Y}\left({ }^{t} \widetilde{S}_{Y^{*}} \widetilde{T}_{Y}\right)
$$

and the second relation follows from ${ }^{t} S_{X^{*}}=\widetilde{S}_{Y^{*}}$ and ${ }^{t} T_{X}=\widetilde{T}_{Y}$ together with the neutrality axiom of Frobenius duality.

We are now in a position to state the coherence theorem for Frobenius reciprocity.

We first prepare some formal definitions. Let $\mathscr{C}$ be strict tensor category with duality. By a signed object in $\mathscr{C}$, we shall mean a pair $(X, \epsilon)$, where $X$ is an object in $\mathscr{C}$ and $\epsilon \in\{ \pm\}$. A signed word in $\mathscr{C}$ is, by definition, a finite sequence of signed objects in $\mathscr{C}$. Two words can be composed by juxtaposition as usual.

Given a signed word $w=\left(\left(X_{1}, \epsilon_{1}\right), \ldots,\left(X_{n}, \epsilon_{n}\right)\right)$, we set $w^{*}=\left(\left(X_{n},-\epsilon_{n}\right)\right.$, $\left.\ldots,\left(X_{1},-\epsilon_{1}\right)\right)$, which is again a signed word, and define an object $[w]$ in $\mathscr{C}$ by

$$
[w]=X_{1}^{\epsilon_{1}} \otimes \ldots \otimes X_{n}^{\epsilon_{n}},
$$

where $X^{\epsilon}=X$ or $X^{*}$ according to $\epsilon=+$ or $\epsilon=-$.

In the totality of pairs of signed words, we introduce the structure of unoriented graph by assigning three types of edges attributed by $L, R$ and $T$ respectively so that

(i) $\left(w_{1} w_{2}, w_{3}\right)$ and $\left(w_{2}, w_{1}^{*} w_{3}\right)$ are jointed by an $L$-edge. 
(ii) $\left(w_{1} w_{2}, w_{3}\right)$ and $\left(w_{1}, w_{3} w_{2}^{*}\right)$ are jointed by an $R$-edge.

(iii) $\left(w_{1}, w_{2}\right)$ and $\left(w_{2}^{*}, w_{1}^{*}\right)$ are joined by a $T$-edge.

Given a path $\gamma$, i.e., a consecutive sequence of edges, from $\left(w_{1}, w_{2}\right)$ to $\left(w_{3}, w_{4}\right)$, we define an isomorphism $[\gamma]: \operatorname{Hom}\left(\left[w_{1}\right],\left[w_{2}\right]\right) \rightarrow \operatorname{Hom}\left(\left[w_{3}\right],\left[w_{4}\right]\right)$ of vector spaces as the repeated composition of Frobenius transforms and transpositions according to the attribution of edges, i.e, $L$ - and $R$-edges correspond to left and right Frobenius transforms respectively while $T$-edges are replaced by transpositions.

THEOREM 3.9. Repeated compositions of transposed maps, Frobenius transforms and their inverses give the same result whenever the initial and the final hom-sets are the same.

More precisely, let $\gamma$ and $\gamma^{\prime}$ be two paths from a pair $\left(w_{1}, w_{2}\right)$ of signed words to another pair $\left(w_{3}, w_{4}\right)$ of signed words. Then they give rise to the same isomorphism, i.e., $[\gamma]=\left[\gamma^{\prime}\right]$.

Proof. Since the formal account requires much space, main steps in the proof are presented below.

The coherence theorem $[\gamma]=\left[\gamma^{\prime}\right]$ is equivalent to have $\left[\gamma^{-1} \gamma^{\prime}\right]=$ id on $\operatorname{Hom}\left(\left[w_{1}\right],\left[w_{2}\right]\right)$. Thus, by taking the Frobenius transform of the form $\operatorname{Hom}(V, W) \rightarrow H\left(V^{*} W\right)$, the problem is reduced to show $\gamma=\mathrm{id}$ on a cyclic tensor product $\operatorname{Hom}\left(I,\left[w_{1}^{*} w_{2}\right]\right)$ if $\gamma$ is a closed path starting and ending at the pair $\left(\emptyset, w_{1}^{*} w_{2}\right)$.

To be explicit, let $[\gamma]$ be given by

$$
H\left(X_{1} \ldots X_{n}\right) \rightarrow \operatorname{Hom}\left(Y_{1}, Z_{1}\right) \rightarrow \ldots \rightarrow \operatorname{Hom}\left(Y_{m}, Z_{m}\right) \rightarrow H\left(X_{1} \ldots X_{n}\right),
$$

where each step is a single Frobenius transform or a transposed map. Then, by the iteration lemma for Frobenius transforms and the definition of cyclic transform, we obtain the commutative diagram

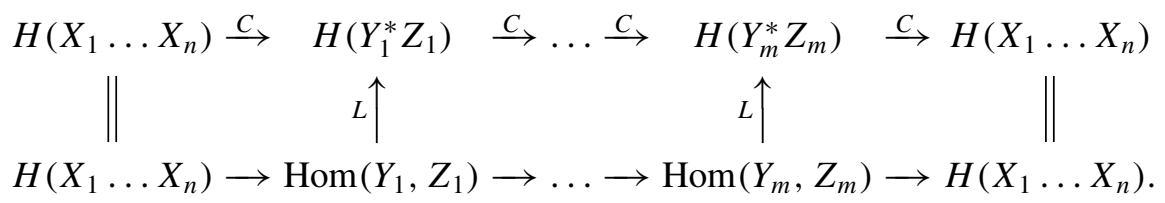

Since the upper line in the diagram turns out to be an iteration of cyclic transforms, the cyclic symmetry shows that it is reduced to the identity and we are done.

REMARK. In the theorem, we need to distinguish $X$ with its dual $X^{*}$ formally: Even if there is a canonical isomorphism between $X$ and $X^{*}$, like $X=Y \oplus Y^{*}$, 
we should not identify $X$ with $X^{*}$. Otherwise, we cannot discriminate $f$ with ${ }^{t} f$ for example.

\section{Self-Duality and Positivity}

Inner product spaces like Hilbert spaces have the self-duality in the sense that dual spaces can be canonically identified with conjugate spaces. This fact is reflected in the existence of ${ }^{*}$-operations on the space of morphisms (i.e., linear operators).

In this section, we shall introduce an analogous structure in tensor categories.

Definition 4.1. A *-tensor category is a tensor category with conjugatelinear involutions $*: \operatorname{Hom}(X, Y) \rightarrow \operatorname{Hom}(Y, X)$ satisfying (i) $(S T)^{*}=$ $T^{*} S^{*}$, (ii) ${ }^{t}\left(T^{*}\right)=\left({ }^{t} T\right)^{*}$ for $S \in \operatorname{Hom}(Y, Z), T \in \operatorname{Hom}(X, Y)$, and (iii) $(S \otimes T)^{*}=S^{*} \otimes T^{*}$ for $S \in \operatorname{End}(X), T \in \operatorname{End}(Y)$.

In a ${ }^{*}$-tensor category, the operation ${ }^{t} T^{*}$ is denoted by $\bar{T}$ and called the conjugation of $T$. In accordance with this terminology, dual objects are also referred to as conjugate objects.

REMARK. In case of non-strict tensor categories, we need to require the unitarity of constraints for the definition of *-tensor categories.

ExAmPLE 4.2. By the obvious *-operations on the set of bounded linear operators in Hilbert spaces, the tensor categories in Example 1.2 and Example 1.4 are *-tensor categories.

Example 4.3. In Example 1.3, assume that $\mathscr{M}$ is a Hopf*-algebra: the underlying algebra structure of $\mathscr{M}$ is provided with a ${ }^{*}$-operation satisfying the compatibility condition $\sigma(x)^{*}=\sigma^{-1}\left(x^{*}\right)$. Moreover, assume that the element $h \in \mathscr{M}$ is self-adjoint with respect to this ${ }^{*}$-operation. The associated transposed map is then ${ }^{*}$-preserving. By a ${ }^{*}$-representation of $\mathscr{M}$, we mean a ${ }^{*}$ representation of $\mathscr{M}$ in a finite-dimensional (possibly indefinite) inner product space. The category $\mathscr{R}^{*}(G)$ of finite-dimensional *-representations of $\mathscr{M}$ is then a *-category as a subcategory of $\mathscr{R}(G)$ with the obvious *-operations on Hom-sets and we can even consider the smaller tensor category $\mathscr{U} \mathscr{R}(G)$ consisting of ${ }^{*}$-representations in finite dimensional Hilbert spaces, the unitary Tannaka dual of $G$.

Definition 4.4. Let $\epsilon=\left\{\epsilon_{X}\right\}$ be a Frobenius duality in a ${ }^{*}$-tensor category $\mathscr{C}$. Then the family $\bar{\epsilon}=\left\{\bar{\epsilon}_{X}\right\}$ also satisfies the axioms of Frobenius duality and is called the conjugation of $\epsilon$. The family $\left\{\epsilon_{X}\right\}$ is said to be self-conjugate if $\overline{\epsilon_{X}}=\epsilon_{X}$ for any object $X$. 
Example 4.5. The Frobenius duality in Example 2.1 is self-conjugate. The Frobenius duality in Example 2.2 is also self-conjugate when it is restricted to the subcategory $\mathscr{R}^{*}(G)$.

The following is immediate from the fact ${ }^{t} \bar{\epsilon}_{X}=\epsilon_{X}^{*}$.

Lemma 4.6. For a self-conjugate Frobenius duality, the associated trace in Lemma 2.6 is * ${ }^{*}$ preserving, i.e., $\langle f\rangle_{X}^{*}=\left\langle f^{*}\right\rangle_{X}$.

For a self-conjugate Frobenius duality, define a (possibly degenerate) End $(I)$-valued hermitian inner product in $\operatorname{Hom}(X, Y)$ by

$$
(S \mid T)=\left\langle S^{*} T\right\rangle_{X}, \quad S, T \in \operatorname{Hom}(X, Y) .
$$

Lemma 4.7. For a self-conjugate Frobenius duality, Frobenius transforms preserve inner products.

Proof. For the left transform $L: \operatorname{Hom}(X, Y) \rightarrow H\left(X^{*} \otimes Y\right)$, this is checked by

$$
\begin{aligned}
L(T)^{*} L(T) & =\epsilon_{X^{*}}\left(1_{X^{*}} \otimes T^{*} T\right) \epsilon_{X^{*}}^{*}=\epsilon_{X^{*}}\left({ }^{t}\left(T^{*} T\right) \otimes 1_{X}\right) \epsilon_{X^{*}}^{*} \\
& =\tau_{X^{*}}\left({ }^{t}\left(T^{*} T\right)\right)=\tau_{X}\left(T^{*} T\right) .
\end{aligned}
$$

Similarly for the right duality map.

Definition 4.8. Let $\mathscr{C}$ be a ${ }^{*}$-tensor category with the simple unit object. A self-conjugate Frobenius duality in $\mathscr{C}$ is positive (resp. positive definite) if the inner products introduced above are positive semidefinite (resp. positive definite). Given a positive Frobenius duality, we put

$$
\operatorname{Ker}(X, Y)=\left\{T \in \operatorname{Hom}(X, Y) ;\left\langle T^{*} T\right\rangle_{X}=0\right\} .
$$

Lemma 4.9.

(i) For $T \in \operatorname{Ker}(X, Y), T^{*} \in \operatorname{Ker}(Y, X)$ and ${ }^{t} T \in \operatorname{Ker}\left(Y^{*}, X^{*}\right)$.

(ii) For $T \in \operatorname{Ker}(X, Y)$ and $S \in \operatorname{Hom}(Y, Z)$, $S T \in \operatorname{Ker}(X, Z)$.

Proof. (i) follows from Lemma 2.7 while (ii) is ensured by Cauchy-Schwartz inequality.

Given a *-tensor category $\mathscr{C}$ with a positive Frobenius duality, we define the quotient category $\overline{\mathscr{C}}$ as follows: The objects in $\overline{\mathscr{C}}$ consists of objects $X$ in $\mathscr{C}$ such that $d(X)=0$. We denote objects in $\overline{\mathscr{C}}$ as $\bar{X}$ to distinguish them from objects in $\mathscr{C}$. The Hom-sets are defined to be

$$
\operatorname{Hom}(\bar{X}, \bar{Y})=\operatorname{Hom}(X, Y) / \operatorname{Ker}(X, Y) .
$$


By the positivity of Frobenius duality, the right hand side is a semi-simple quotient and operations of tensor product and conjugation as well as *-operation are brought down to the quotient category by the above lemma. The tensor category $\overline{\mathscr{C}}$ also inherits the Frobenius duality.

In this way, we have obtained the quotient *-tesnor category with positive definite Frobenius duality and the study of tensor categories of this type is more or less reduced to the positive definite case.

Proposition 4.10. Let $\mathscr{C}$ be $a^{*}$-tensor category with a positive definite Frobenius duality. Then the following holds.

(i) For a non-zero object $X$ in $\mathscr{C}, d(X) \geq 1$.

(ii) The hermitian inner product introduced before Lemma 4.8 is positive definite and Frobenius transforms are unitary.

(iii) Cyclic transforms are self-conjugate in the sense that

$$
\overline{C_{X, Y}(\bar{T})}=C_{X, Y}(T)
$$

for $T \in H(X \otimes Y)$.

REMARK. Cyclic symmetry can be geometrically interpreted as $6 j$-symbols as pointed out in [22] and the key identities for $6 j$-symbols derived there (cf. also [4]) for the *-tensor category of bimodules of $I I_{1}$-factors can be easily extended to the present context as long as positivity is ensured for the relevant Frobenius duality.

In the remaining, we shall examine the procedure discussed in $\S 4$ by a simple but fundamental example; the Tannaka dual of $S L_{q}(2, \mathrm{C})$ with $q$ a root of unity. Since most of computations are repetitions of [12]. we will just indicate the points where we need to take cares.

Recall that the quantum group $S L_{q}(2, \mathrm{C})$ is described by the algebra $U_{q}$ generated by $\{k, e, f\}$ with the relation

$k k^{-1}=1=k^{-1} k, \quad$ ef $-f e=\frac{k^{2}-k^{-2}}{q-q^{-1}}, \quad k e k^{-1}=q e, \quad k f k^{-1}=q^{-1} f$

together with the coalgebra structure defined by

$$
\begin{gathered}
\Delta(k)=k \otimes k, \quad \Delta(e)=e \otimes k^{-1}+k \otimes e, \\
\Delta(f)=f \otimes k^{-1}+k \otimes f \\
\epsilon(k)=1, \quad \epsilon(e)=0=\epsilon(f) .
\end{gathered}
$$

The antipode $\sigma$ is then given by

$$
\sigma(k)=k^{-1}, \quad \sigma(e)=-q^{-1} e, \quad \sigma(f)=-q f
$$


and $h=k^{-1}$ satisfies the properties in Example 1.3.

Moreover $U_{q}$ with $|q|=1$, is made into a Hopf *-algebra so that

$$
k^{*}=k, \quad e^{*}=e, \quad f^{*}=f .
$$

Note that this is the only *-structure in $U_{q}$ up to isomorphism ([19]), whence the choice of *-operation in [12] is not compatible with the Hopf algebra structure.

Now we assume that $q$ is a primitive $m$-th root of unity $(m \geq 3)$ and exclusively consider finite-dimensional representations of $U_{q}$ for which $e$ and $f$ are representated by nilpotent operators. (The nilpotency is automatic for finite dimensional irreducible representations.) Note that the totality of such representations is closed under taking tensor products and transposed maps.

Standard arguments of highest weight vectors show that any irreducible representation of $U_{q}$ has dimension less than or equal to $m^{\prime}$, where $m^{\prime}$ denotes the minimal positive integer satisfying $q^{m^{\prime}}=q^{-m^{\prime}}$ (i.e., $m^{\prime}=m / 2$ if $m$ is even and $m^{\prime}=m$ otherwise), and the equivalence classes of irreducible representations are parametrized by the dimension $l+1$ of representation and a complex number $\omega$ satisfying $\omega^{4}=q^{2 l}$ for $0 \leq l \leq m^{\prime}-2$ and $\omega^{4} \notin\left\{q^{2 j} ; 0 \leq j \leq m^{\prime}-2\right\}$ for $l=m^{\prime}-1$ ([24]). More precisely, if we denote by $V(l, \omega)$ the corresponding irreducible representation, then $V(l, \omega)$ has a basis of the form $\left\{v, f v, f^{2} v, \cdots, f^{l} v\right\}$ such that $e v=0$ and $k v=\omega v$. Note that $e^{m^{\prime}}=f^{m^{\prime}}=0$ in any of these representations.

As to the duality relation of representations, we have $V(l, \omega)^{*} \cong V\left(l, q^{l} \omega^{-1}\right)$. In fact, if we define a linear functional $v^{*} \in V(l, \omega)^{*}$ by $\left\langle v^{*}, f^{n} v\right\rangle=\delta_{n, 0}$, it satisfies $k v^{*}=\omega^{-1} v^{*}$ and $f v^{*}=0$, i.e., $v^{*}$ is a lowest weight vector in $V(l, \omega)^{*}$. The highest weight vector is then given by $e^{l} v^{*}$ with the highest weight $k e^{l} v^{*}=\omega^{-1} q^{l} v^{*}$.

Proposition 4.11. The irreducible representation $V(l, \omega)$ can be made into $a^{*}$-representation of $U_{q}$ (by providing a suitable hermitian inner product) if and only if $\omega^{2}=q^{l}$ for $0 \leq l \leq m^{\prime}-2$ or $\omega^{2} q^{1-m^{\prime}}>0$ for $l=m^{\prime}-1$.

Proof. Assume that $V(l, \omega)$ admits a non-trivial invariant hermitian inner product $(\mid)$. Since $f$ should be hermitian, the inner product is determined by real numbers $\left(f^{a} v \mid f^{b} v\right)=\left(v \mid f^{a+b} v\right)$ with the obvious relation $\left(v \mid f^{j} v\right)=0$ for $j>l$. By

$$
0=\left(e v \mid f^{j+1} v\right)=\left(v \mid e f^{j+1} v\right)=[j+1]_{q} \frac{\omega^{2} q^{-j}-\omega^{-2} q^{j}}{q-q^{-1}}\left(v \mid f^{j} v\right),
$$

we know that $\left(v \mid f^{j} v\right)=0$ for $0 \leq j<l$. Thus the inner product, if it exists, is unique up to scalar multiple. By comparing $\left(k v \mid f^{l} v\right)$ and $\left(v \mid k f^{l} v\right)$, we have $\bar{\omega}=\omega q^{-l}$. 
Conversely, if $\omega$ satisfies this condition, then it is easy to check that the hermitian inner product in $V$ defined by

$$
\left(f^{a} v \mid f^{b} v\right)= \begin{cases}1 & \text { if } a+b=l, \\ 0 & \text { otherwise }\end{cases}
$$

is invariant under the action of $U_{q}$.

The quantum dimension of these *-representations are given by

$$
q^{l}+q^{l-2}+\cdots+q^{-l}=\frac{q^{l+1}-q^{-l-1}}{q-q^{-1}}=[l+1]_{q}
$$

and the following can be easily checked: $[l+1]_{q}>0$ for $0 \leq l \leq m^{\prime}-2$ if and only if $m$ is even and $q \in\left\{e^{ \pm 2 \pi i / m}\right\}$. (Note that $\left[m^{\prime}\right]_{q}=0$.)

This condition is ovbiously necessary to ensure the positivity of the Frobenius duality in the Tannaka dual of $U_{q}$ and, in fact, it is also sufficient because the quantum trace vanishes on degenerate representations in [12] for such a choice of $q$ :

Proposition 4.12. Let $q$ be a primitive $m$-th root of 1 with $m \geq 3$. Then the Tannaka dual of $U_{q}$ is never positive-definite but it is positive semidefinite if and only if $m$ is even and $q \in\left\{e^{ \pm 2 \pi i / m}\right\}$.

If the positivity condition is satisfied, the equivalence classes of irreducible objects in its positive definite quotient have representatives $V\left(l, \pm q^{l / 2}\right)$.

Since the square root indeterminancy of $\omega$ is not essential for representation theory, we shall choose a square root $q^{1 / 2}$ of $q$ once for all and the irreducible representation $V\left(l,\left(q^{1 / 2}\right)^{l}\right)$ is simply denoted by $V_{l}$ for $0 \leq l \leq d-2$. Note that we have chosen so that $V_{l}^{*} \cong V_{l}$ and the trivial representation $V_{0}$.

Now the following is an immediate consequence of the corresponding fusion rule in the Tannaka dual $\mathscr{C}$ of $S L_{q}(2)$.

Proposition 4.13. Let $q$ be as in the previous proposition and $\mathscr{R}$ be the *tensor category generated by $\left\{V_{l}\right\}_{1 \leq l \leq d-2}$. Then the quotient category $\overline{\mathscr{R}}$ by the kernel ideal of $\mathscr{R}$ is semisimple and the equivalence classes of simple objects are represented by the family $\left\{V_{l}\right\}$ with the fusion rule given by

$$
\bar{V}_{j} \otimes \bar{V}_{k} \cong \begin{cases}\bigoplus_{l=|j-k|,|j-k|+2, \cdots, j+k} \bar{V}_{l} & \text { if } j+k \leq d-2, \\ \bigoplus_{l=|j-k|,|j-k|+2, \cdots, 2(d-2)-j-k} \bar{V}_{l} & \text { otherwise. }\end{cases}
$$

Remark. The tensor category $\overline{\mathscr{C}}$ completely describes that of bimodules associated to subfactors of type $A_{n}$. Note that $\overline{V_{1}}$ is self-conjugate and generates the principa graph of type $A_{d-1}$. 


\section{REFERENCES}

1. Chari, V. and Pressley, A., A Guide to Quantum Groups, Cambridge Univ. Press, 1994.

2. Doplicher, S. and Roberts, J. E., A new duality theory for compact groups, Invent. Math. 98 (1989), 157-218.

3. Durhuus, B., Jakobsen, H. P. and Nest, R., Topological quantum field theories from generalized $6 j$-symbols, Rev. Math. Phys. 5 (1993), 1-67.

4. Evans, D. and Kawahigashi, Y., Quantum Symmetries on Operator Algebras, Oxford Univ. Press, 1998.

5. Fröhlich, J. and Kerler, T., Quantum Groups, Quantum Categories and Quantum Field Theory, Lecture Notes in Math. 1542 (1993).

6. Ghez, P., Lima, R. and Roberts, J., $W^{*}$-categories, Pacific J. Math. 120 (1985), 79-109.

7. Goodman, F. M., de la Harpe, P. and Jones, V., Coxeter-Dynkin Diagrams and Towers of Algebras, Springer-Verlag, 1989.

8. Hiai, F., Minimizing indices of conditional expectations onto a subfactor, Publ. Res. Inst. Math. Sci. 24 (1988), 673-678.

9. Izumi, M., Application of fusion rules to classification of subfactors, Publ. Res. Inst. Math. Sci. 27 (1991), 953-994.

10. Kajiwara, T. and Yamagami, S., Irreducible bimodules associated with crossed product algebras II, Pacific J. Math. 171 (1995), 209-229.

11. Kassel, C., Quantum Groups, Springer-Verlag, Berlin-New York, 1995.

12. Keller, G., Fusion rules of $U_{q}\left(s l\left(2\right.\right.$, C) ), $q^{m}=1$, Lett. Math. Phys. 21 (1991), 273-286.

13. Kosaki, H. and Yamagami, S., Irreducible bimodules associated with crossed product algebras, Internat. J. Math. 3 (1992), 661-676.

14. Longo, R., Index of subfactors and statistics of quantum fields. I, Comm. Math. Phys. 126 (1989), 217-247.

15. Longo, R., Index of subfactors and statistics of quantum fields. II, Comm. Math. Phys. 130 (1990), 285-309.

16. Longo, R. and Roberts, J. E., A theory of dimension, K-Theory 11 (1997), 103-159.

17. MacLane, S., Natural associativity and commutativity, Rice Univ. Stud. 49 (1963), 28-46.

18. MacLane, S., Categories for the Working Mathematician, second ed., Springer-Verlag, NewYork, 1998.

19. Masuda, T., Mimachi, K., Nakagami, Y., Noumi, M., Saburi, Y. and Ueno, K., Unitary representations of quantum $S U_{q}(1,1)$, Lett. Math. Phys. 19 (1990), 187-204.

20. Ocneanu, A., Quantized groups, string algebras, and Galois theory for algebras, Operator algebras and Applications, vol. 2, 1988, Cambridge University Press.

21. Ocneanu, A., Quantum Symmetry, Differential Geometry of Finite Graphs and Classification of Subfactors, University of Tokyo Seminary Notes, 1991.

22. Ocneanu, A., An invariant coupling between 3-manifolds and subfactors with connections to topological and conformal field theory, an abstract of results with appendices and figures, preprint.

23. Popa, S., Classification of amenable subfactors of type II, Acta Math. 172 (1994), 163-255.

24. Roche, P. and Arnaudon, D., Irreducible representations of the quantum analogue of $S U$ (2), Lett. Math. Phys. 17 (1989), 295-300.

25. Saavedra Rivano, N., Catégories Tannakiennes, Lecture Notes in Math. 265 (1972).

26. Sauvageot, J. -L., Sur le prodiut tensoriel relatif d'espaces de hilbert, J. Operator Theory 9 (1983), 237-252.

27. Sunder, V. S. and Vijayarajan, A. K., On the non-occurrence of the Coxeter graphs $D_{2 n+1}, E_{7}$ as principal graphs of an inclusion of II 1 factors, Pacific J. Math. 161 (1993), 185-200.

28. Turaev, V. G., Quantum Invariants of Knots and 3-Manifolds, de Gruyter, Berlin-New York, 1994. 
29. Turaev, V. and Viro, O., State sum invariants of 3-manifolds and quantum 6j-symbols, Topology 31 (1992), 865-902.

30. Yamagami, S., A note on Ocneanu's approach to Jones index theory, Internat. J. Math. 4 (1993), 859-871.

31. Yamagami, S., Modular theory for bimodules, J. Funct. Anal. 125 (1994), 327-357.

32. Yamagami, S., On unitary representation theories of compact quantum groups, Comm. Math. Phys. 167 (1995), 509-529.

33. Yamagami, S., Crossed products in bimodules, Math. Ann. 305 (1996), 1-24.

34. Yamagami, S., On Ocneanu's characterization of crossed products, preprint.

35. Yamagami, S., Group symmetry in tensor categories, preprint.

DEPARTMENT OF MATHEMATICAL SCIENCES

IBARAKI UNIVERSITY

MITO, 310-8512

JAPAN 